\title{
LOS GRÁFICOS CARTESIANOS RELATIVOS A CIRCUITOS ELÉCTRICOS DE CORRIENTE ALTERNA PRESENTES EN LIBROS DE TEXTO UNIVERSITARIOS
}

Norah Giacosa, Ramiro Galeano, Claudia Zang, Alejandro Such y Carlos Puglisi (*)

Resumen: Se muestran resultados de un estudio descriptivo de casos múltiples. Se examinaron, mediante el análisis de contenido, los gráficos cartesianos existentes en diez libros de texto universitarios de uso frecuente en Argentina durante la presentación del tema circuitos eléctricos de corriente alterna. Se encontraron deficiencias que fomentarían errores conceptuales y omisiones que dificultarían la comprensión lectora por parte de quienes consultan estos ejemplares. Se concluye que las imprecisiones señaladas obstaculizarían la construcción de aprendizajes significativos.

Palabras claves: gráfico cartesiano, circuito eléctrico, corriente alterna, libro de texto.

\section{Introducción}

Los estudiantes universitarios de carreras científico-tecnológicas tienen dificultades para comprender los fenómenos que acontecen en circuitos de corriente alterna (CA). Si bien son múltiples los factores que podrían señalarse, los autores sostienen que uno de ellos está relacionado con los libros de texto (LT) que utilizan.

Los LT son una modalidad específica de recurso didáctico diseñado para el acto pedagógico de un nivel educativo particular. Desde esta perspectiva, son considerados herramientas mediadoras que, ajustándose a los lineamientos curriculares oficiales de un contexto específico, responden a principios didáctico-pedagógicos (Occelli y Valeiras, 2013). A diferencia de otros materiales (enciclopedias, revistas de difusión científica, etc.) se presume que la lectura debe realizarse en el orden propuesto por los autores para el logro de determinados aprendizajes (Campanario, 2003).

Por otro lado, se sostiene que la comprensión lectora no es la mera decodificación literal del texto en sí, sino más bien la construcción de significados que elabora el lector basándose en sus experiencias previas, intereses, esquemas cognitivos y propósitos pretendidos. Es sabido que los LT de Física entrañan una doble dificultad, por un lado usan un lenguaje especializado, donde el significado de algunos términos difiere en ocasiones del que se le atribuye en la vida cotidiana; por otro, utilizan, de una manera particular por su intensidad y extensión, las Matemáticas como herramienta privilegiada para describir los fenómenos físicos. Al respecto, Alexander y Kulicowich (citados en Pocoví y Ovejero, 2009) consideran que

(*) Departamento de Física. Facultad de Ciencias Exactas, Químicas y Naturales. UNaM. Félix de Azara 1552. (3300) Posadas. Misiones.norah@correo.unam.edu.ar 
los LT de Física son "bilingües", pues utilizan alternativamente un sistema lingüístico (expresiones verbales) y un sistema simbólico (ecuaciones, gráficos, diagramas, etc.) entre los cuales el lector debe desplazarse para lograr la comprensión. Cuanto más breves o implícitas sean las traducciones, mayores serán los requerimientos de procesamiento de información que deberá poner en juego el lector.

El propósito de este trabajo es presentar resultados derivados del análisis de los gráficos cartesianos (GC), relativos a circuitos de CA, presentes en diez LT universitarios de uso frecuente en Argentina.

La gráfica, es una representación visual elaborada a partir de un conjunto de datos y basada en la noción de función matemática. Su principal objetivo es poner de manifiesto, utilizando un conjunto de convenciones y formalismos, la relación que guardan entre sí los datos; lo cual posibilita analizar un proceso o un fenómeno. Como posee un alto nivel de abstracción, su lectura es más compleja que la de otras representaciones, por ejemplo esquemas o dibujos; y requie- re por parte del lector, de un dominio de códigos específicos para su decodificación. Los GC, aquellos que utilizan como sistema de referencia ejes ortogonales entre sí, constituyen una parte medular del circuito comunicativo en Ciencias y del trabajo experimental.

Por otro lado, existen numerosos reportes de investigaciones en torno a los LT que se utilizan en las aulas en distintos niveles educativos formales, realizadas desde distintas perspectivas y con diferentes propósitos. Algunos de los ellos dan cuenta de la presentación de ciertos tópicos de Física presentes en los LT universitarios. De la búsqueda bibliográfica realizada surge que, las investigaciones que analizaron GC (García, 2005; García y Perales, 2009), no tuvieron por objeto de estudio los GC relativos a circuitos eléctricos de CA.

\section{Aspectos metodológicos}

La metodología empleada en esta investigación se sitúa en la modalidad de estudio descriptivo de casos múltiples (AnderEgg, 2010). Se seleccionó un conjunto de

\begin{tabular}{|l|l|}
\hline Código & Libro de texto \\
\hline T1 & $\begin{array}{l}\text { Alonso, E. y Finn, E. (1976) Fisica. Vol. II Campos y ondas. Barcelona. España: Fondo Educativo } \\
\text { Interamericano S.A. }\end{array}$ \\
\hline T2 & $\begin{array}{l}\text { Bauer, W. y Westfall, G. (2011) Física para Ingeniería y Ciencias con Física moderna. Volumen } 2 \text {. }^{\circ} \text { Ed. } \\
\text { México: McGraw Hill. }\end{array}$ \\
\hline T3 & $\begin{array}{l}\text { Gettys, E.; Keller, F. y Skove, M. (2005) Física para Ciencias e Ingeniería. Tomo II. México: McGraw } \\
\text { Hill. }\end{array}$ \\
\hline T4 & $\begin{array}{l}\text { Giancoli, D. (2009) Física para Ciencias e Ingeniería con Fisica moderna. Volumen II. 4ta Ed. México: } \\
\text { Pearson Educación }\end{array}$ \\
\hline T5 & $\begin{array}{l}\text { Halliday, D.; Resnick, R. y Krane, K. (1999). Física. Vol. 2. 4ta. Ed. México: Compañia Ed. Continental, } \\
\text { SA. }\end{array}$ \\
\hline T6 & $\begin{array}{l}\text { McKelvey, J. y Grotch, H. (1981) Física para ciencias e ingenieria. Tomo II. Ira. Ed. México: Harla } \\
\text { S.A. }\end{array}$ \\
\hline T7 & $\begin{array}{l}\text { Serway, R. y Jewett, J. (2009) Fisica para ciencia e ingenieria con Fisica Moderna. Vol. 2. 7ma. } \\
\text { México: Ed. Cenage Learning Editores S.A. }\end{array}$ \\
\hline T8 & Tipler, P. (1993) Física. Tomo 2. 3ra Ed. España: Editorial Reverté S.A. \\
\hline T9 & Tippens, P. (2011) Física, conceptos y aplicaciones. $7^{\circ}$ Ed. Perú:McGraw Hill. \\
\hline T10 & $\begin{array}{l}\text { Young, H. y Freedman, R. (2009) Fisica universitaria con Fisica Moderna. Vol.2. 12 }{ }^{\circ} \text { Ed. México: } \\
\text { Pearson Educación. }\end{array}$ \\
\hline
\end{tabular}

Tabla $\mathbf{N}^{0}$ 1: Libros de texto y código asignado 
LT universitarios que se utilizan frecuentemente para la enseñanza de Electromagnetismo en el ciclo básico de carreras científico-tecnológicas que se desarrollan en Argentina. Seguidamente, dada la imposibilidad de analizar todos los LT existentes en el mercado, se escogieron diez de ellos y se codificaron como se muestra en la Tabla No 1 . Dicho código se usará de aquí en más para referirse a ellos.

El material impreso fue examinado mediante el análisis de contenido (Bardín, 1996). Las categorías que se usaron tuvieron en cuenta los siguientes aspectos: modelos representados, uso didáctico, uso científico, identificación explícita de variables independiente y dependiente que se representan, tiempos para los cuales se grafican las cantidades alternantes, señalamientos sobre los ejes de coordenadas, amplitudes y argumentos de las funciones temporales de $\mathrm{CA}$ y voltaje.

\section{Discusión de resultados}

En la muestra examinada existen 34 gráficos cartesianos de corriente y voltaje alterno de circuitos simples ( $\mathrm{R}, \mathrm{L}$ o $\mathrm{C}) \mathrm{y}$ combinados (RLC en serie). En 30 ocasiones se representan ambas cantidades alternantes en el mismo sistema de referencia. En un solo LT, T7, se presenta una figura (33.13.b) que contiene 4 gráficos temporales de CA y diferencia de potencial en los elementos pasivos del circuito. Dicha figura es conceptualmente incorrecta porque contradice las hipótesis iniciales planteadas por los autores (, al ignorar el ángulo de fase. En la muestra examinada prevalecen representaciones basadas en modelos teóricos generales, sin asignación de valores numéricos a las variables (33/34). El único ejemplo numérico se presenta en T10 y forma parte de la resolución dada a uno de los problemas resueltos. En idéntica proporción, el uso didáctico es expositivo, es decir se utilizan GC como parte de la descripción de fenómenos temporales que acontecen en circuitos de CA, existiendo un solo GC que tiene uso problemático. Todos los GC examinados tienen un uso científico teórico, en ninguna ocasión el GC representa un grupo determinado de datos experimentales.

Del análisis realizado en torno a la identificación explícita de la variable independiente surge que en el $21 \%$ de los GC, es decir $7 / 34$, se omite dicha referencia. Las 7 figuras se presentan en T3 $(31.8 ; 31.10$; $31.12 ; 31.17)$ y T9 $(32.7 ; 32.8 ; 32.9)$. En el $50 \%$ de casos estudiados no se identifica explícitamente la variable dependiente. Los 17 GC se muestran en $\mathrm{T} 1$ (17.25; 17.26), T2 (30.8; 30.10; 30.12; 30.17.a, 30.17.b, 30.17.c), T3 (31.8; 31.10; 31.12; 31.17), T5 (3.b, 4.b, 5.b) у T8 $(28.5 ; 28.7)$.

En la mayoría de los LT que utilizan como referencia al tiempo, no se realizan señalamientos sobre el eje de la abscisa (T2, $\mathrm{T} 4, \mathrm{~T} 8, \mathrm{~T} 10)$, pero en T1 uno de los tiempos se expresa como cociente entre un ángulo y la frecuencia angular $(\mathbb{\nabla} / \mathbb{\nabla})$; y en otro (T7) se indica un solo tiempo, el período de las funciones oscilantes. En los dos LT que utilizan ángulo variable con el tiempo $(T 3, T 5)$ se señalan $\pi$ y $2 \pi$; y en uno de ellos (T3) además, se marca un valor genérico con la notación " $\bigotimes t$ t". En el único LT que no se indica la variable independiente (T9) se señala sobre el eje ciertos valores de ángulos $(0,90,180,270,360)$ medidos en grados sexagesimales. Esto último, al entender 
de los autores de la investigación, es inapropiado. En el 60\% de los LT (T1,T2,T3,T4, T5, T8) se omite referencia del eje de las ordenadas, no obstante, en tres de ellos (T2, T3, T5) se señalan sobre él los valores máximos de CA y de voltaje. En tres LT (T7, T9, T10) se indica que en dicho eje se representa la CA y la diferencia de potencial, pero sólo en uno, T7, se especifica además con un subíndice si la diferencia de potencial y la CA corresponden a una resistencia, un inductor o un condensador.

En todos los LT se representan las funciones de voltaje y $\mathrm{CA}$ en un sistema de ejes ortogonales, lo cual supone aceptar que se considera como origen del tiempo a la intersección de los ejes. No obstante, en los GC se pueden distinguir tres criterios diferentes. En algunos LT (T3, T4, T7, T8) se grafican ambas funciones únicamente para tiempos iguales o superiores a cero. En otros (T2, T5, T10), se representan ambas funciones para tiempos "anteriores" al momento en el que se puso en marcha el cronómetro que posibilita estudiar el fenómeno y; en los restantes (T1, T9), las funciones se representan para tiempos disímiles, una de ellas para valores de tiempos iguales o mayores a cero y la otra, solamente para tiempo mayores a cero. Estos últimos GC son, al parecer de los autores, conceptualmente incorrectos, porque para determinados intervalos de tiempo no se grafica la CA ni el voltaje. Los 3 casos identificados se exhiben en T1 (17.26) y T9 $(32.8 ; 32.9)$.
Respecto a las amplitudes de las funciones graficadas, es de hacer notar que sólo en T10 se indica explícitamente que las escalas para representar a la corriente y el voltaje son distintas, de manera que las amplitudes de las curvas no son significativas. Examinando los argumentos de las funciones oscilantes, se aprecia que existen 2 ejemplares que muestran una suma de cantidades dimensionalmente incompatibles en los GC. Así, en T4 (30.16.b; 30.17.b) y T10 (30.17.b; 30.19.b) se incluyen leyendas del tipo o , las cuales fomentarían errores difíciles de erradicar en los lectores que consultan estos LT.

\section{Conclusiones}

Las omisiones señaladas, aunque parezcan triviales, representan dificultades para los estudiantes y obstaculizan la comprensión lectora. Paralelamente, algunas deficiencias citadas, tales como sumar cantidades dimensionalmente incompatibles fomentarían errores conceptuales, difíciles de erradicar en quienes consultan estos ejemplares.

Dadas las características del estudio exploratorio de casos múltiples descrito, los resultados obtenidos no son, obviamente, generalizables. Sin embargo, se espera que los mismos puedan servir de referencia para orientar a los docentes de Física en el proceso de selección y recomendación de LT. 


\section{Referencias}

Ander-Egg, E. (2010) Métodos y Técnicas de investigación social, Vol. III: Cómo organizar el trabajo de investigación España: Lumen.

Bardín, L. (1996) El análisis de contenido. Madrid: Akal.

Campanario, J. (2003) De la necesidad a la virtud: cómo aprovechar los errores e imprecisiones de los libros de texto para enseñar Física. Revista Enseñanza de la Ciencias. (21) 1; p.161-172

García J. (2005). El uso y el volumen de información en las representaciones gráficas cartesianas presentadas en los libros de texto de ciencias experimentales. Enseñanza de las Ciencias, 23(2), p.181-200.

García, J. y Perales, F. (2009). ¿Atribuyen los profesores de ciencias las mismas características y usos a las gráficas cartesianas que los autores de los textos? Revista Educación y Pedagogía, Vol. XVII, No43, p. 81-89.

Occelli, M. y Valeiras, N. (2013) Los libros de texto de ciencias como objeto de investigación: una revisión bibliográfica. Enseñanza de las Ciencias, 31(2), p.133-152.

Pocoví, M. y Ovejero, A. (2009) Aprendizaje de la noción de aceleración angular a partir de textos. Memorias de las XVI Reunión Nacional de Educación en Física. San Juan, Argentina. Universidad Nacional de Cuyo. CD-ROM. 